\title{
ALPVEN SOLITONS IN THE SOLAR WIND
}

\author{
Chris ovenden and steven J. Schwart $z^{*}$ \\ Department of Applied Mathenatics \\ Queen Mary College \\ vile End Road \\ Iondon El 4NS \\ UR
}

\section{ABSTRACT}

We present here a nonlinear Alfven soliton solution of the MHD equations. This solution may represent the final state of modulationally unstable Alfven waves. We also briefly describe a model of the expected turbulent spectrum due to a collection of such solitons.

${ }^{*}$ Currently a Nuffield Foundation Science Research Fellow

1. Introduction

The Alfvenic nature of the large amplitude turbulence in the solar wind has been known for some time (Belcher and Davis 1971). The relationship between this turbulence and traditional linear MrD waves or special large amplitude solutions (Barnes and Hollweg 1974; Abraham-Shrauner and Feldman 1977) remains unclear. Equally unclear are the consequences this turbulence may have for solar wind particle populations, although ion drift speeds proportional to the Alfven speed and high perpendicular temperatures suggest some wave-particle connections (Schwartz et al. 1981).

Efforts to describe solar wind turbulence usually begin with a perturbation expansion, leading either to the "decay cascade" (Chin and Wentzel 1972; Cohen and Dewar 1974; Schwartz 1977) if the system is described purely in terms of its linear modes, or to the modulational instability (Derby 1978; Goldstein 1978) of a single large amplitude Alfven wave. We discuss here a third alternative which leads to a nonlinear soliton solution. This solution may represent the state to which a modulationally unstable Alfven wave relaxes. Using an ensemble average of collections of these solitons, we find a turbulence power spectrum $\epsilon(k) \alpha_{k}-2$ at high frequencies which flattens toward the low frequency end. Details of the calculations can be found in ovenden et al. (1983). Further work on alternative turbulence models and particle-soliton interactions is currently in progress.

\section{The Soliton Solution}

We begin with the one fluid description of a plasma: continuity and momentum equations and the generalized Ohm's law coupled with Maxwell's equations (eg. Boyd and Sanderson $19693.40,3.54$ and 3.613.63). Assuming quasi-neutrality, an isothermal equation of state, and neglecting the displacement current, resistivity and the $\partial_{j} / \partial t$ 
term in the generalized Ohm's law, it is easy to eliminate the electric field and transverse fluid velocity. For simplicity we consider only parallel-propagation so that $\partial / \partial x=0=\partial / \partial y$ where the $z$ direction corresponds to that of the background magnetic field Bo. Ultimately, the resulting equations can be written

$$
\begin{gathered}
\frac{\partial^{2} B_{ \pm}}{\partial t^{2}}-\frac{\partial}{\partial z}\left[\frac{v_{A}^{2}}{n} \frac{\partial}{\partial z} B_{ \pm}\right]+\frac{\partial}{\partial z}\left[v \frac{\partial B_{ \pm}}{\partial t}+\frac{d}{d t}\left(v B_{ \pm}\right)\right] \\
\pm \frac{i}{\Omega_{i}} v_{A}^{2} \frac{\partial}{\partial z}\left[\frac{d}{d t}\left[\frac{1}{n} \frac{\partial B_{ \pm}}{\partial z}\right]\right]=0
\end{gathered}
$$

and

$$
\left[\frac{\partial^{2}}{\partial t^{2}}-c_{B}^{2} \frac{\partial^{2}}{\partial z^{2}}\right]_{n}=\frac{v_{A}^{2}}{2} \frac{\partial^{2}}{\partial z^{2}}\left(\left|B_{ \pm}\right|^{2}\right)+\frac{\partial^{2}}{\partial z^{2}}\left(n v^{2}\right)
$$

where $B_{ \pm}=B_{x} \pm i B_{y}, \quad v$ is the z-component of the fluid velocity, $n$ is the density, $v_{A}=B_{O} / \sqrt{ }(4 \pi \rho)$ is the Alfven speed, $\Omega_{i}$ the proton gyrofrequency, $c_{s}$ the isothermal sound speed, and $d / d t=\partial / \partial t+v \partial / \partial z$. Both $B_{ \pm}$and $n$ have been normalized to their background values ( $B_{0}$ and $n_{0}$ respectively). The harmonic solutions to (1.1) and (1.2) with $\left|B_{ \pm}\right|$ independent of $z$ are jugt the Alfven-Ion cyclotron modes investigated by Abraham-Shrauner and Feldman (1977).

and we are interested in modes with small but nonzero fluctuations on linearize (1.1) and (1.2) with respect to these quantities. Fuxthermore, we insert an Alfven wave with slowly varying amplitude, viz.,

$$
B_{ \pm} \quad=b(z, t) e^{i\left(k_{A} z-\omega_{O \pm} t\right)}
$$

where $\omega_{O \pm}=\omega_{A}\left(I \mp \omega_{A} / 2 \Omega_{i}\right)$ and assuming $\omega_{A}<<\Omega_{i}$ with $\omega_{A}=k_{A} v_{A}$. At this point, the modulational instability calculation proceeds by perturbing the $b=$ constant solution with fluctuations $o b, \quad o n, v$ all varying harmonically in space and time. Such a calculation yields instabilities with e-folding lengths $\approx 10^{-2} \mathrm{AU}$.

Here, following zakharov (1972) we search instead for soliton solutions of (1.1)-(1.2) with a wave amplitude in (1.3) given by

$$
b(z, t)=b_{0} \operatorname{sech}[k(z-v t)] e^{i \delta \omega t}
$$

keeping terms to third order in $|\mathrm{b}|$ and assuming $b$ varies slowly 
compared to the original Alfven wave. This process yields selfconsistent constants $v, K$, and $\delta w$ given by

$$
\begin{aligned}
v & \approx v_{A}\left[1-\frac{\omega_{A}}{\Omega_{i}}\right] \\
\kappa & =\frac{k_{A} b_{o}}{2}\left[\frac{\Omega_{i} / \omega_{A}}{1-\beta}\right]^{1 / 2}
\end{aligned}
$$

and

$$
\delta \omega=-\frac{\omega_{A}\left|b_{O}\right|^{2}}{8(1-B)}
$$

for values of the ratio of sound to Alfven speed squared $(\beta)<<1$ and for the $\omega_{\text {ot }}$ (left hand polarized) wave. The density (in nondimensional units) and velocity variations are given by

$$
\text { on }=\frac{|b(z, t)|^{2}}{2(1-\beta)}
$$

and

$$
\mathbf{v}=\mathbf{v} \text { ôn }
$$

Physically, the increased density (1.6) leads to a lower local Alfven speed causing the associated build up in wave energy in this region. The energy transport is achieved by forcing this material to move at the wave group velocity $(1.5 \mathrm{a})$, thereby resulting in the unchanged soliton envelope. It is not yet clear whether the solution (1.4)-(1.9) represents a true soliton, in the senge that two guch solutions would pass through one another without interacting.

\section{Turbulence Spectrum}

To build a picture of a turbulent spectrum out of solitons, we follow the work of Kingsep et al. (1973) and Yu and spatschek (1976) who consider an ensemble of systems with a common energy density $w$ (normalized to $\mathrm{B}_{\mathrm{O}} 2 / 8 \pi$ ) and length $\mathrm{L}$. The various realizations correspond to different numbers, $N$, of non-overlapping, assumed identical, solitons into which this energy is divided. The value of $N$ lieg in the interval

$$
1 \leqslant N \leqslant K L
$$

Ensemble averaging over these realizations, which we take with equal probablities, yields an energy spectrum

$$
\left\langle\left|b_{k}\right|^{2}\right\rangle \propto \frac{1}{(\sigma k)^{2}}[\sigma k \tanh (\sigma k)-\log \cosh (\sigma k)]
$$

where

$$
\sigma=\frac{\pi}{k_{A}}\left[\frac{\omega_{A}(1-\beta)}{\Omega_{i} 8 \pi W}\right]^{1 / 2}
$$


As can be seen in the plot of (2.1) shown in Fig. 1 (or directly from the explicit form of (2.1)), this spectrum is flat at low frequencies and falls off steeply as $\mathbf{k}^{-2}$ at high frequencies. Although this is qualitatively similar to solar wind observations (eg. Bavassano et al. 1982; Denskat and Neubauer 1982 ), it is not yet clear whether quantitative agreement can be improved within the soliton description by, eg., an alternative ensemble average, collections of unequal solitons, etc.

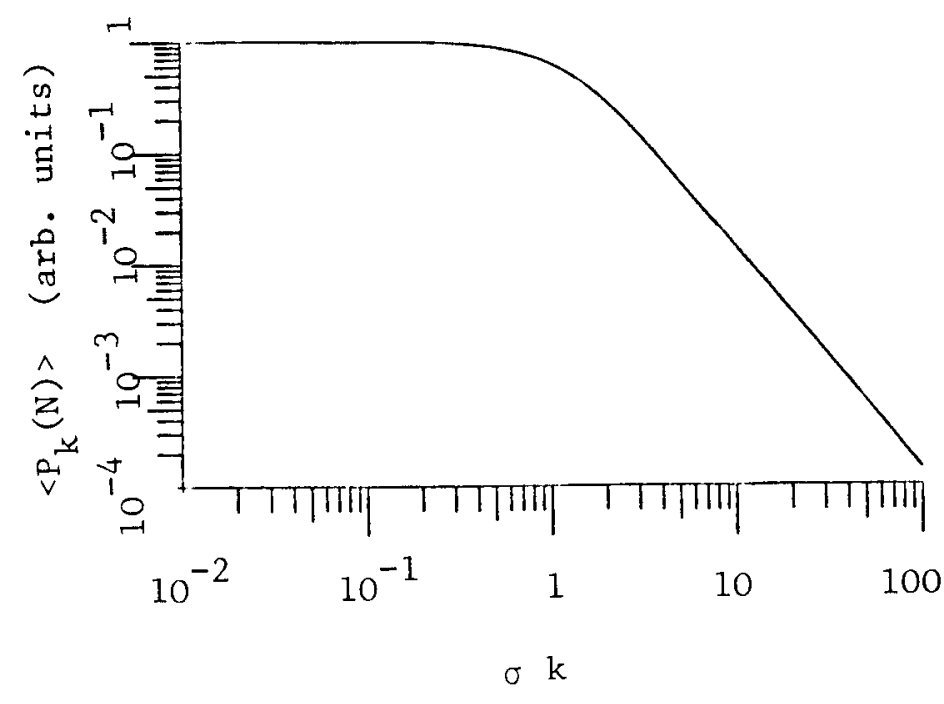

Fig. 1. Ensemble averaged power spectrum of various collections of solitons describing the same total turbulent energy.

3. Summary envisage as the final state of a modulationally unstable Alfven wave . Dividing turbulent energy in a given region into such solitons and averaging over the different possible arrangements yields a turbulent spectrum which is flat at low $k$ and falls as $k^{-2}$ at large $k$. Remaining questions concern other possible descriptions of solar wind turbulence within this context and the consequences of particle-soliton interactions for solar wind ion distributions.

Acknolweagements

We are grateful to Hassan shah for many detailed suggestions.
This work was supported by the science and Engineering Research Council via grant SG/D 04442 .

\section{References}

Abraham-shrauner, B. and W.C. Feldman: Nonlinear Alfven waves in high-speed solar wind streams, J. Geophys. Res. 82, 618, 1977.

Barnes, A. and J.V. Hollweg: Large-amplitude hydromagnetic waves, J. Geophys. Res. 79, 2302, 1974.

Bavagsano, B., M. Dobrowolny, F. Mariani and N.F. Negs: Radial evolution of power spectra of interplanetary Alfven turbulence, J. Geophys. Res. 87, 3617, 1982 . 
Belcher, J.W. and I. Davis Jr.: Large-amplitude Alfven waves in the interplanetary medium, 2, J. Geophys. Res. 76, 3534, 1971.

Boyd, T.J. and J.J. Sanderson: Plasma dynamics, Thomas Nelson \& Sons Ltd., London, 1969.

Chin, Y. and D.G. Wentzel: Nonlinear dissipation of Alfven waves, Astrophys. Sp. Sci. 16, 465, 1972.

Cohen, R.H. and R.I. Dewar: on the backscatter instability of solar wind Alfven waves, J. Geophys. Res. 79, 4174, 1974.

Denskat, K. and F.M. Neubauer: statistical properties of low frequency magnetic field fluctuations in the solar wind from 0.29 to 1.0 AU during solar minimum conditions: HELIOS $I$ and II, J. Geophys. Re8. 87, 2215, 1982.

Derby, N.F. Jr.: Modulational instability of finite-amplitude, circularly polarized Alfven waves, Astrophys. J. 224, 1013, 1978.

Goldstein, M.L.: An instability of finite amplitude circularly polarized Alfven waves, Astrophys. J. 219, 700, 1978.

Kingsep, A.S., C.I. Rudakov and R.N. Sudan: Spectra of strong Langmuir turbulence, Phys. Rev. Lett. 31, 1482, 1973.

Ovenden, C.R., H. Shah and S.J. Schwartz: Alfven solitons in the solar wind, J. Geophys. Res., in press, 1983.

schwartz, S.J.: Cosmic ray generated anisotropic magnetohydrodynamic turbulence, Kon. Not. R. Astr. Soc. 178, 399, 1977.

Schwartz, S.J., W.C. Feldman and S.P. Gary: The source of proton anisotropy in the high speed solar wind, J. Geophys. Res. 86, $541,1981$.

YU, M.Y. and K.M. Spatschek: Anomalous damping of ion-acoustic waves in the presence of strong Langmuir turbulence, Phys. Fl. 19, 705, 1976,

Zakharov, V.E.: Collapse of Langmuir waves, Sov. Phys. J.E.T.P. 35, $908,1972$. 